\title{
Psychiatric Comorbidity as a Risk Factor for Mortality in People with Anorexia Nervosa
}

\author{
1 Department of Psychological Medicine, King's College London, London, UK \\ 2 South London and Maudsley NHS Foundation Trust, London, UK \\ ${ }^{3}$ Department of Health and Welfare, University of Taipei, Taipei City, Taiwan
}

Hubertus Himmerich ${ }^{1,2}$, Matthew Hotopf ${ }^{1,2}$, Hitesh Shetty ${ }^{2}$, Ulrike Schmidt ${ }^{1,2}$, Janet Treasure $^{1,2}$, Richard D. Hayes ${ }^{1}$, Robert Stewart ${ }^{1,2}$ and Chin-Kuo Chang $^{1,2,3^{*}}$

\section{Abstract}

Anorexia nervosa (AN) is found associated with increased mortality. Frequent comorbidities of AN include substance use disorders (SUD), affective disorders (AD) and personality comorbidities on all-cause mortality with demographic and socioeconomic factors considered as confounders in the 1,970 people with AN, using data from the case register of the South London and Maudsley (SLaM) NHS Foundation Trust, an almost monopoly secondary mental healthcare service provider in southeast London. We retrieved data from its Clinical Records Interactive Search (CRIS) system as data source. Mortality was ascertained through nationwide tracing by the UK Office for National Statistics (ONS) linked to CRIS database on a monthly basis. A total of 43 people with AN died during the observation period. Standardized Mortality Ratio (SMR) with England and Wales population in 2012 as standard population for our study comorbidity of SUD or PD was found to significantly increase the relative risks of mortality $(\mathrm{HRs}=3.10,95 \% \mathrm{Cl}: 1.21,7.92$; and $2.58,95 \% \mathrm{Cl}: 1.23,5.40$, respectively). After adjustment for demographic and socioeconomic covariates as confounders, moderately but not significantly elevated risks were identified for SUD (adjusted HR=1.39, 95\% Cl: 0.53, 3.65) and PD (adjusted $\mathrm{HR}=1.58,95 \% \mathrm{Cl}: 0.70,3.56)$. These results suggest an elevated mortality in people with $\mathrm{AN}$, which might be, at least partially, explained by the existence of the comorbidities SUD or PD.

\section{Background}

- Anorexia nervosa (AN, ICD-10: F50.0/F50.1): a significantly low body weight in the context of age, sex, and physical health, intense fear of weight gain and disturbed body perception, with prevalence between $<1 \%$ and $4 \%$ among women, and a M:F ratio of $1: 10$

- The most common causes of death in AN are sudden cardiac death associated with ventricular arrhythmias, and suicide. Mortality rates are 5-6 times higher for people with AN than in the general population.

- AN is often accompanied by other mental disorders, including substance use disorders (SUD, F10-F19), affective disorders (AD, F31-F33), and personality disorders (PD, F60). disorders (PD). We investigated the influence of these psychiatric observation window between January 2007 and March 2016 for cohort was 5.21 (95\% Cl: 3.77, 7.02). In univariable analyses, the
Aim

- To investigate the influence of major psychiatric comorbidities on all-cause mortality in people with AN

\section{Methods}

- Study design: a retrospective cohort study (Jan 2007 - Mar 2016)

- Setting: case registry in South London and Maudsley NHS Foundation Trust (SLaM), providing near-monopoly secondary mental healthcare services to a catchment of about 1.36 million residents in southeast London

- Data source: anonymised electronic clinical records in SLaM, using the Clinical Record Interactive Search (CRIS) system

- Mortality identification: UK mortality registry from the ONS with data linkage to SLaM by NHS number

- Statistical analysis: Standardized Mortality Ratio (SMR) was used for adjusting age and gender, with the population of England and Wales in 2012 as the standard population, with specific weightings applied by the average follow-up period for corresponding ageand-sex bands. And, Cox proportional hazards regression was used to estimate the hazard ratio $(\mathrm{HR})$ for each of the psychiatric comorbidity, with age, sex, ethnicity, borough, marital status and deprivation score considered as confounders.

\section{Results}

- A total of 1,970 AN subjects were identified with a mean age of 24.5 years old $(S D=11.3)$ and 43 of them died in follow-up period.

- SMR for the AN cohort $=5.21(95 \% \mathrm{Cl}: 3.77,7.02)$

- Univariable analysis revealing a significant effect of age, marital status, deprivation score, SUD and PD (partially shown in Table 1)

- Multivariable analysis showing significant moderately elevated risks for SUD (adjusted $\mathrm{HR}=1.39,95 \% \mathrm{Cl}: 0.53,3.65$ ) and PD (adjusted $\mathrm{HR}=1.58,95 \% \mathrm{Cl}: 0.70,3.56$ ), when controlling for potential confounders (detailed in Table 2)

\section{Conclusion \& Discussion}

- Summary: 43 of 1,970 people with AN died during the observation period of more than nine years of follow-up.

- A more than five folds of general mortality found by age and gender standardization, compared to the general population in England and Wales.

- Moderately elevated mortality risks estimated for comorbid SUD and PD with confounders controlled, albeit not significant.

- Strengths: cohort study design; precise mortality identification

- Limitations: A short follow-up period, resulting in only 43 deaths observed and limited statistical power; generalizability to secondary and tertiary health care settings only
Table 1. Univariable analysis on mortality among people with people with anorexia nervosa by Cox regressions $(N=1,970)$
Table 2. Multivariable analysis on mortality among people with anorexia nervosa by Cox regressions, $(N=1,970)^{\wedge}$

\begin{tabular}{|c|c|c|c|c|c|c|c|}
\hline & $\begin{array}{l}\text { Mean } \pm \text { SD / } \\
\text { Numbers } \\
\text { (\% in column) }\end{array}$ & $\begin{array}{l}\text { Number of } \\
\text { deaths } \\
\text { (\% in row) }\end{array}$ & $\begin{array}{l}\text { Hazard Ratio } \\
(95 \% \mathrm{CI})\end{array}$ & $\begin{array}{l}p- \\
\text { valu }\end{array}$ & & $\begin{array}{l}\text { Adjusted Hazard } \\
\text { Ratio }(95 \% \mathrm{Cl}) \\
080(0.36181)\end{array}$ & $\frac{p \text {-value }}{060}$ \\
\hline Substance us & & & & & \multirow{2}{*}{$\begin{array}{l}\text { Affective disorder } \\
\text { Substance use } \\
\text { disorder }\end{array}$} & & \\
\hline No & $1,909(96.90 \%)$ & 38 (1.99\%) & Ref & & & $3,3.65)$ & 0.50 \\
\hline Yes & $61(3.10 \%)$ & $5(8.20 \%)$ & $3.10(1.21,7.92)^{*}$ & $<0.05$ & Personality disorder & $1.58(0.70,3.56)$ & 0.27 \\
\hline
\end{tabular}

No

Yes

Personality disorder

No

Yes
Affective disorder
$1,680(85.28 \%) \quad 34(2.02 \%) \quad$ Ref $290(14.72 \%) \quad 9(3.10 \%) \quad 1.40(0.67,2.93)$ $\wedge$ Controlling age, sex, ethnicity, borough, marital status and deprivation score
Corresponding to Dr. Chin-Kuo Chang, Department of Health and Welfare, University of Taipei, Taipei City,

Taiwan; e-mail address: ckchang@utaipei.edu.tw 